\title{
rIL-22 as an adjuvant enhances the immunogenicity of $r$ GroEL in mice and its protective efficacy against $S$. Typhi and $S$. Typhimurium
}

Gurpreet Kaur, Chitradevi, Charu and Anju Bansal

Corrigendum to: Cellular \& Molecular Immunology (2015) 12, 96-106; doi:10.1038/cmi.2014.34; published online 24 February 2016

In this article, published online 26 May 2014, the names of two of the authors were spelled incorrectly. The names should have been listed above as Gurpreet Kaur, Chitradevi STS, Charu Nimker and Anju Bansal. The authors regret the errors. 